\title{
Electrical and compositional properties on Bridgman-grown Gd-doped GaSb substrates
}

\author{
J.L. Plaza ${ }^{\mathrm{a}, *}$, P. Hidalgo ${ }^{\mathrm{b}}$, B. Méndez ${ }^{\mathrm{b}}$, J. Piqueras ${ }^{\mathrm{b}}$, J.L. Castaño ${ }^{\mathrm{c}}$, E. Diéguez ${ }^{\mathrm{a}}$ \\ a Departamento de Física de Materiales, Universidad Autónoma de Madrid 28049, Cantoblanco, Madrid, Spain \\ ${ }^{\mathrm{b}}$ Departamento de Física de Materiales, Universidad Complutense 28040, Madrid, Spain \\ c Departamento de Física de Aplicada, Universidad Autónoma de Madrid 28049, Cantoblanco, Madrid, Spain
}

\begin{abstract}
In this work pure and Gd-doped GaSb ingots with different dopant concentrations were grown by the Bridgman Method. The compositional and electrical analysis carried out on these materials have shown the dependence of these results on the initial dopant concentration. The analysis have revealed the presence of inclusions which have been identified as Gd-Sb precipitates. The doped material was found to be p-type with a maximum value of the carrier density at the central region of the ingots. It has been also demonstrated that the Gd enhances the A band emission reducing the band-gap emission. (C) 2001 Elsevier Science B.V. All rights reserved.
\end{abstract}

Keywords: Gallium antimonide; Rare earths; Band-gap

\section{Introduction}

Rare earth (RE) doped semiconductors are promising materials in the field of the optoelectronics and for their potential applications in optical communication systems. The interest in RE doped semiconductors is based on the fabrication of new electroluminescence devices which can combine both the luminescence of $\mathrm{RE}$ ions and the electronic properties of semiconductors [1].

Due to the incomplete $4 \mathrm{f}$ shell of the RE elements which is shielded by the outermost filled $5 s^{2}$ and $5 p^{6}$ orbitals, these elements exhibit atomic-like optical transitions when they are introduced as trivalent ions in solids. These intra- $4 \mathrm{f}$ transitions are parity forbidden in the free ion. On the other hand, due to the mixing of opposite parity states produced by the local electric fields in the solid host [2], these transitions are allowed when the RE ions are embedded in solids. However, the interaction between the RE ions and the matrix is weak and the wavelength of intra- $4 \mathrm{f}$ shell emission is only slightly dependent on the host material and the environmental temperature.

\footnotetext{
* Corresponding author. Tel.: +34-91-3974784; fax: +34-913978579.

E-mail address: jl.plaza@uam.es (J.L. Plaza).
}

The incorporation of several RE ions into III-V semiconductors has been reported in the literature [39]. In particular the Gd has been recently studied due to several good properties when is combined with III-V semiconductors like the passivation of GaAs surfaces $[10,11]$ and for the attainment of a low interfacial density of states in MOS diodes [12].

Among the III-V semiconductors, gallium antimonide $(\mathrm{GaSb})$ is an interesting material due to their applications as substrate for the development of optoelectronic devices with a band-gap emission in the range from 1.1 to $3.9 \mu \mathrm{m}$ being useful for applications in optical fiber communication systems [13]. In this work the effect of the Gd into vertical Bridgman-grown $\mathrm{GaSb}$ has been investigated by means of scanning electron microscope (SEM), energy dispersive $X$ ray analysis (EDX), van der Pauw technique and cathodoluminescence $(\mathrm{CL})$.

\section{Experimental procedure}

The vertical Bridgman technique was used to grow pure (A) and Gd-doped GaSb (B and C) ingots. High purity $99.9999 \% \mathrm{Ga}, \mathrm{Sb}$ and $99.9 \% \mathrm{Gd}$ metal were used as starting material. As crucibles, high quality quartz 

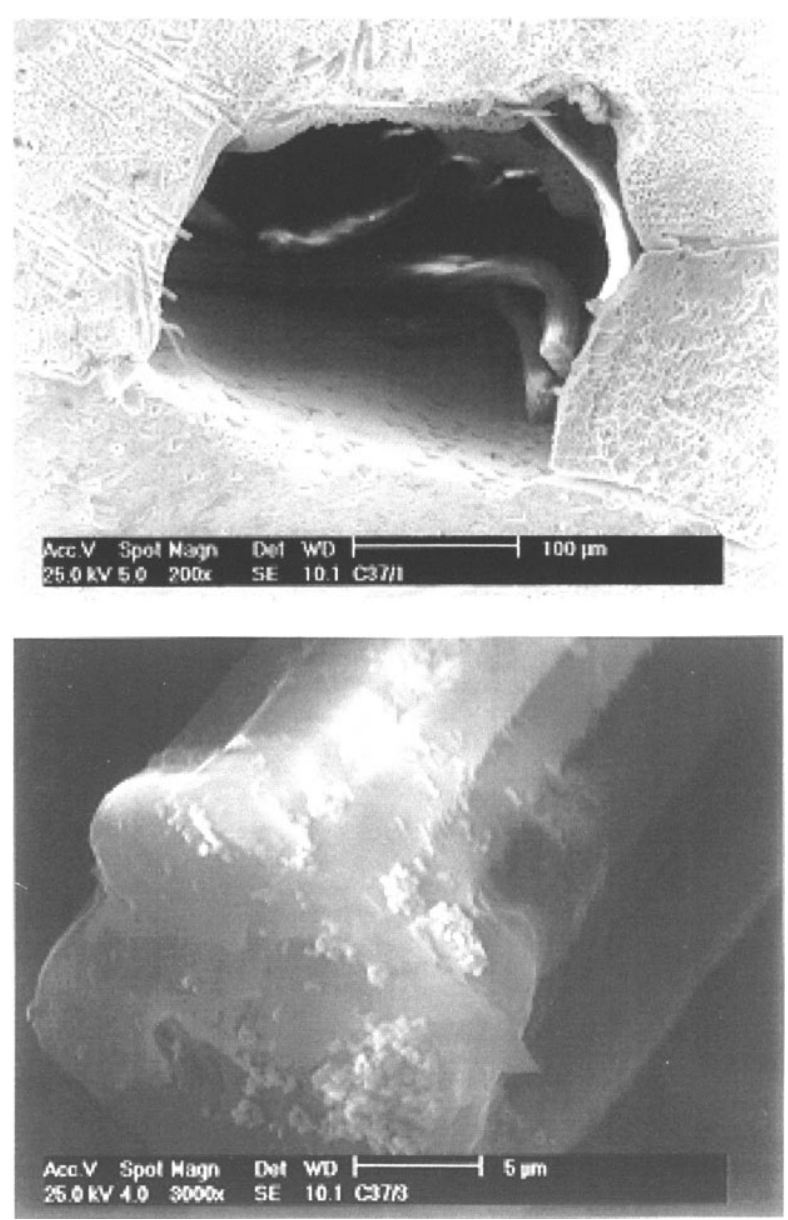

Fig. 1. (a) SEM image from a small hole found in the samples obtained from the last solidified region of the two Gd-doped GaSb ingots. (b) Magnification of a precipitate located inside the holes.

ampoules sealed at $10^{-6}$ Torr were used. The ampoules were also graphited by acetone pirolysis at $1000{ }^{\circ} \mathrm{C}$ in order to prevent the material sticking to the walls. The growth was carried out at a rate of $3 \mathrm{~mm} \mathrm{~h}^{-1}$ in a single-zone oscillatory Bridgman furnace having a temperature gradient of $57^{\circ} \mathrm{C} \mathrm{cm}-1$. More details about the growth process can be found elsewhere [14].

Two different $\mathrm{GaSb}$ ingots were grown with initial Gd concentration in the melt of $2 \times 10^{19} \mathrm{~cm}^{-3}$ (Ingot B) and $8 \times 10^{19} \mathrm{~cm}^{-3}$ (Ingot C) respectively. All of them were $60 \mathrm{~mm}$ long and $12 \mathrm{~mm}$ in diameter. In order to make an analysis of the evolution of the several properties of the material along the growth process, several wafers were obtained along the ingot. All of them were cut perpendicular to the growth direction.

Mechanical polishing was carried out using 5, 1, 0.3 and $0.05 \mu \mathrm{m}$ alumina powder. After this process, chemical etching was applied for 30 seconds with a CP4 type solution $\left(1 \mathrm{CH}_{3} \mathrm{COOH}: 5 \mathrm{HF}: 9 \mathrm{HNO}_{3}: 10 \mathrm{H}_{2} \mathrm{O}\right)$ followed by methanol rinsing. The Van der Pauw technique at room temperature was used for the measurement of resistivity, mobility and carrier density with magnetic fields perpendicular to the samples up to $7 \mathrm{KGauss}$. Indium dots were used as ohmic contacts, which were previously verified. Secondary electron microscope (SEM) images and energy dispersive X-ray analysis (EDX) were carried out with a Philips apparatus model XL30 coupled with an EDX analyser model DX4i. The accelerating voltages were $25 \mathrm{kV}$. The cathodoluminescence (CL) data were obtained with a Hitachi S-2500 SEM at $77 \mathrm{~K}$ at $25 \mathrm{kV}$ as accelerating voltages.

\section{Results and discussion}

All the samples were analysed by SEM and EDAX. No main differences were detected in the samples obtained from the centre and the initial region of the ingots in comparison with the samples extracted from the pure $\mathrm{GaSb}$ ingot. Nevertheless some little holes with

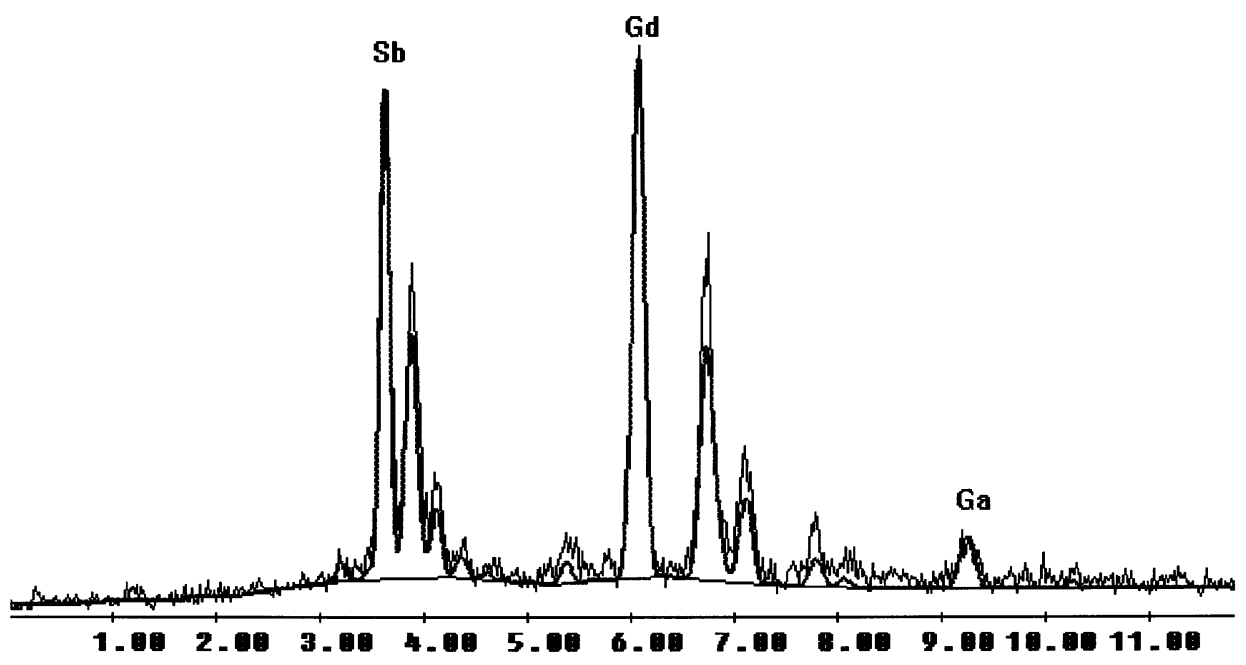

Fig. 2. EDX spectrum obtained from the inclusions present at the final regions of the Gd-doped GaSb ingots. 

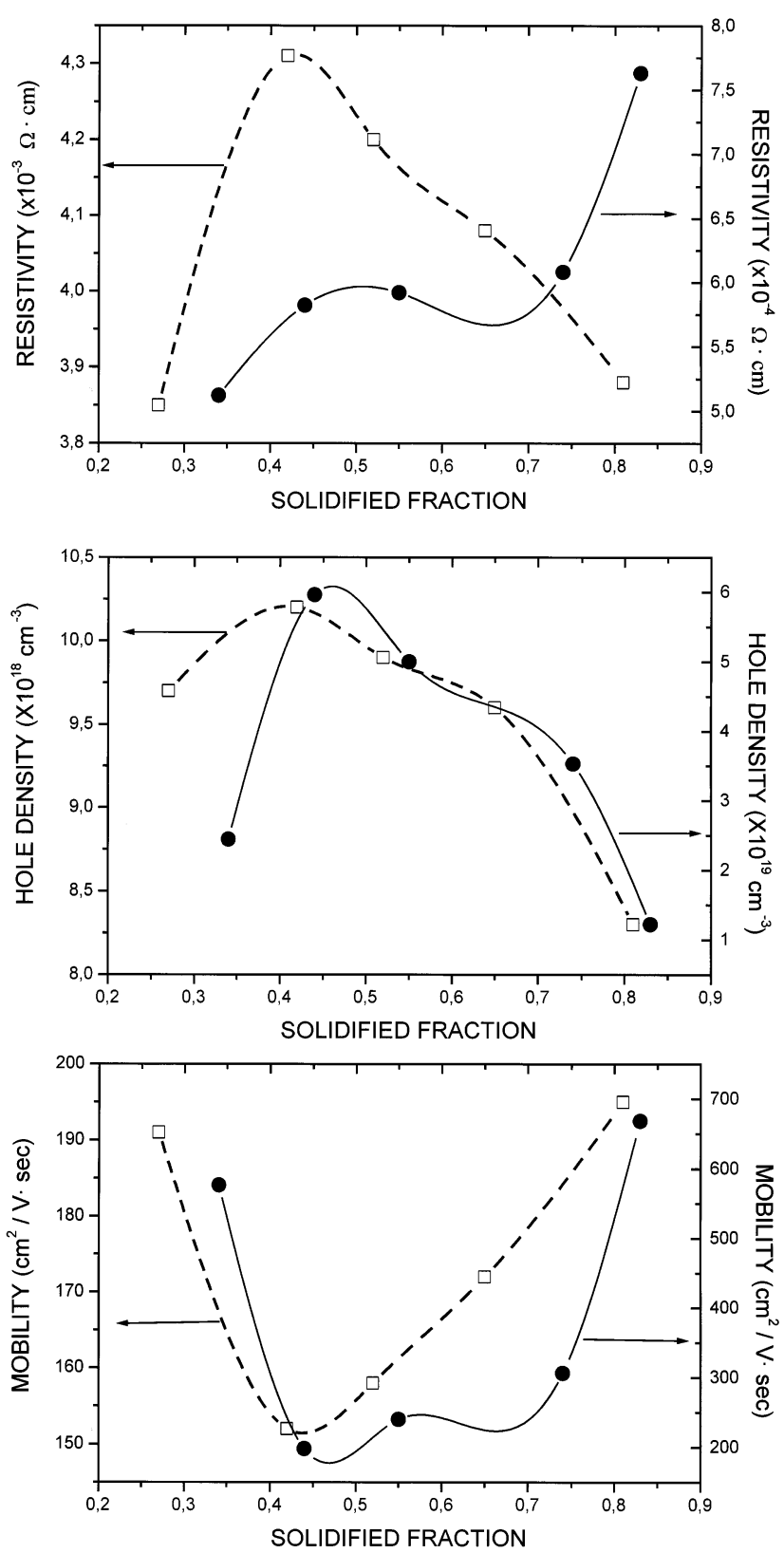

Fig. 3. (a) Resistivity, (b) hole density and (c) mobility at room temperature vs. the solidified fraction for the Gd-doped GaSb. Open squares refer to ingot $\mathrm{B}$ and closed circles to ingot $\mathrm{C}$

small precipitates inside were detected in the last solidified region. A micrograph of these holes found in the two Gd doped GaSb ingots is presented in Fig. 1a.

In order to know the composition of these precipitates, an EDX analysis was carried out. It shows that these precipitates have the following composition: $\mathrm{Sb}$ (37.09 at.\%), Ga (11.08 at.\%) and Gd (51.83 at.\%). The corresponding EDX spectrum is given in Fig. 2. These kind of precipitates whose compositions are mainly $\mathrm{Sb}$ and Gd have been previously reported for other RE dopants like $\mathrm{Er}$ and $\mathrm{Nd}$ in $\mathrm{GaSb}[8,9]$. These compounds are known as pnictides.
Resistivity, mobility and carrier density were obtained by the van der Pauw technique as function of the solidified fraction as shown in Fig. 3.

From the resistivity measurements it can be observed that in the case of the ingot with low Gd concentration (ingot B) a maximum in the resistivity appears in the central region of the ingot. The difference between the values of the resistivity at the centre and the region corresponding to the final stages of the growth process is around $10 \%$. In the case of the ingot with the highest dopant concentration (ingot $\mathrm{C}$ ) there exist an increase in the resistivity along the growth direction. This be-
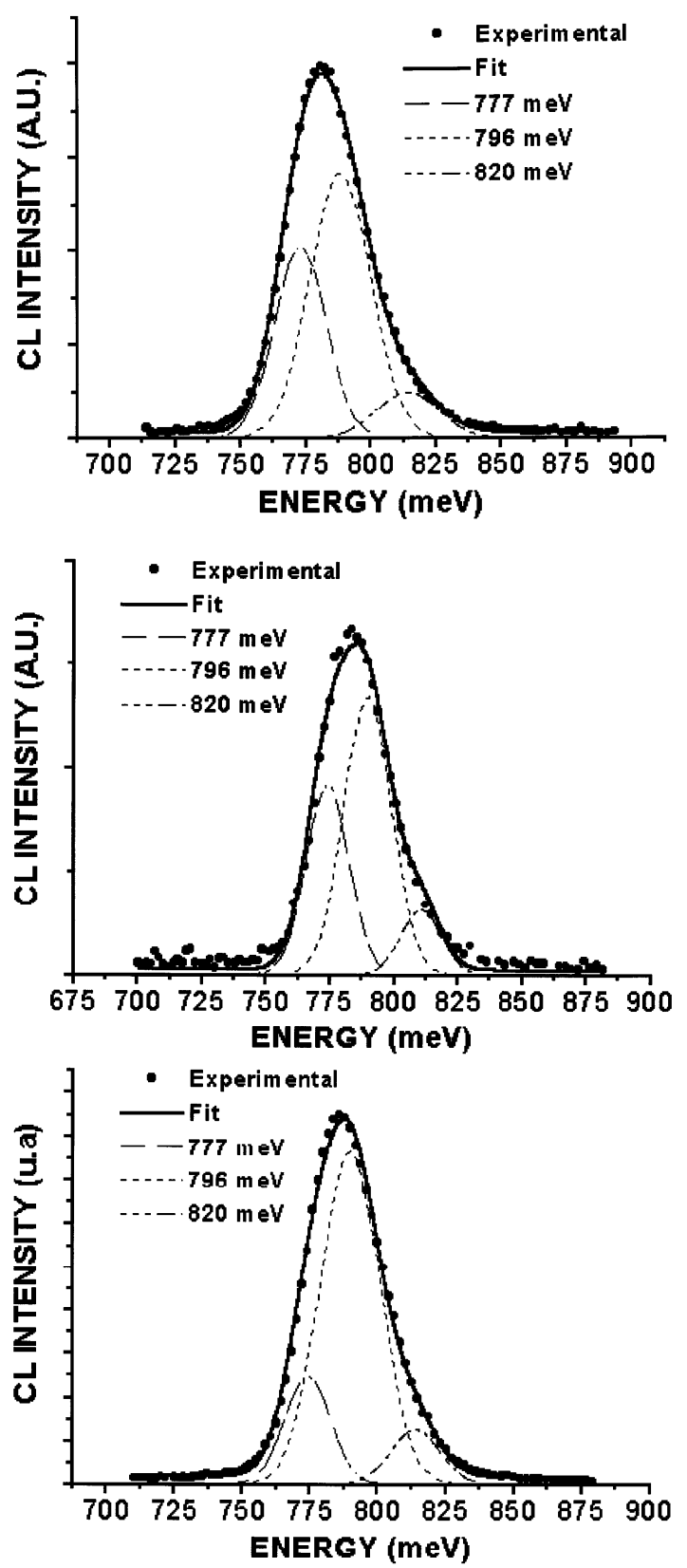

Fig. 4. The CL spectra at $78 \mathrm{~K}$ for three samples along the ingot $\mathrm{B}$. (a) At the initial solidified fraction, (b) at the centre and (c) at the final solidified fraction. 


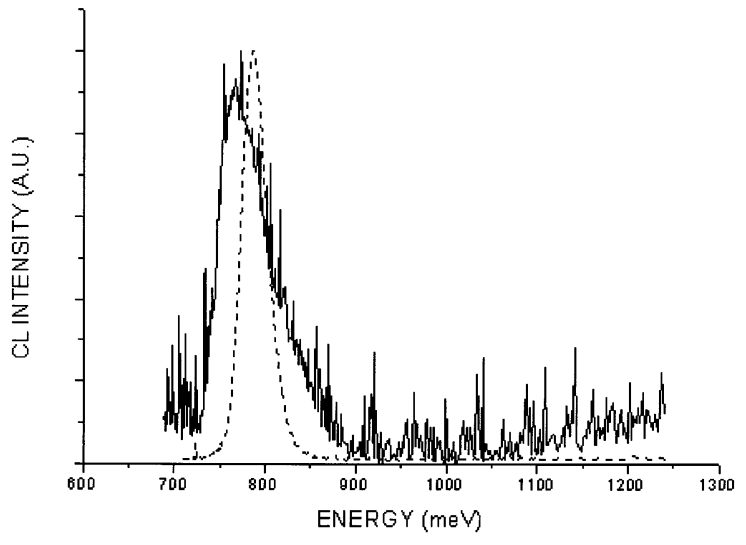

Fig. 5. Comparison of the CL spectra at $78 \mathrm{~K}$ from samples obtained from the ingots $\mathrm{B}$ and $\mathrm{C}$ (the later is the noisiest and is represented by a continuous line).

haviour could indicate that, for the high dopant concentration, the defect density is higher at the final solidified fraction.

Regarding to the hole density (Fig. 3b) it can be observed that, in the two cases, the maximum is placed near the region corresponding to the beginning of the solidification process. This behaviour could indicate that there exists a slightly higher concentration of dopant in this region, as it will be shown below from the $\mathrm{CL}$ analysis. The mobility data presented in Fig. 3c show that the maximum values appear near the top and bottom of the ingots probably due to a lower carrier concentration in these regions. For comparison, the values obtained for the resistivity, carrier density and mobility from the pure $\mathrm{GaSb}$ (ingot $\mathrm{A}$ ) have been obtained being; $0.090 \mathrm{~W} \cdot \mathrm{cm}, 1.0 \times 10^{17} \mathrm{~cm}^{-3}$ and 550 $\mathrm{cm}^{2} / \mathrm{V} \cdot \mathrm{sec}$ respectively.

The CL analysis has been carried out in different samples in the two ingots. Fig. 4 shows the CL spectra for three samples along the ingot $\mathrm{B}$ at the initial solidified fraction (Fig. 4a) at the centre (Fig. 4b) and at the region corresponding to the final stages of the solidification process (Fig. 4c).

From these measurements it can be pointed out that there exists a $\mathrm{Gd}$ gradient along the samples. This gradient is going from low concentration, in samples obtained from the final region of the ingots, to high concentrations at the initial stages of the growth. The CL spectra confirms this feature regarding to the intensity relation between the emissions centred at $773 \mathrm{meV}$ (A Band) and the emissions related to $795 \mathrm{meV}$ (bandgap). It can be seen that the A band increases at the regions at the centre and at the initial values of the solidified fraction. The band related to $815 \mathrm{meV}$ is due to the tail-states and has been observed in other samples. Regarding to the highly doped sample it can be seen that the signal is very low compared with the signal obtained from the samples from ingot B. As can be observed from the noisy spectra presented in Fig. 5. There exist a small displacement of the spectra from samples $\mathrm{C}$ regarding with samples from ingot $\mathrm{A}$. This could confirm that the Gd enhances the A band emission reducing the band-gap emission.

\section{Conclusions}

The effect of the Gd dopant on GaSb Bridgman grown ingots has been investigated. The SEM-EDX analysis indicates the presence of precipitates consisting mainly in $\mathrm{Gd}$ and $\mathrm{Sb}$. The Hall measurements indicate a maximum hole concentration near the region corresponding to the beginning of the solidification process. The CL analysis show that the Gd enhances the emission of the A band decreasing the emission of the band-gap.

\section{Acknowledgements}

This work has been supported by CICYT under the project ESP-98-1340.

\section{References}

[1] S. Coffa, A. Polman, R.N. Schwartz, Rare Earth Doped Semiconductors II, MRS Symp. Proc. 422, Materials Research Society, Pittsburg, 1996.

[2] A.R. Zanatta, L.A.O. Nunes, Appl. Phys. Lett. 71 (25) (1997) 3679.

[3] L.F. Zakharenkov, V.A. Kasatkin, F.P. Kesamanly, B.E. Samorukov, M.A. Sokolova, Sov. Phys. Semicond. 15 (1981) 946.

[4] V.A. Kasatkin, F.P. Kesamanly, V.G. Makarenkov, V.F. Masterov, B.E. Samorukov, Sov. Phys. Semicond. 14 (1980) 1092.

[5] V.A. Kasatkin, F.P. Kesamanly, B.E. Samorukov, Sov. Phys. Semicond. 15 (1981) 352.

[6] H. Ennen, J. Schneider, J. Electron. Mater. 14A (1985) 115.

[7] P. Hidalgo, B. Méndez, J. Piqueras, J. Plaza, E. Diéguez, Semic. Sci. Technol. 13 (1998) 1431.

[8] J.L. Plaza, P. Hidalgo, B. Méndez, J. Piqueras, J.L. Castaño, E. Diéguez, J. Cryst. Growth 198/199 (1999) 379.

[9] J.L. Plaza, P. Hidalgo, B. Méndez, J. Piqueras, E. Diéguez, Mat. Sci. Eng. B71 (2000) 282.

[10] M. Hong, M. Passlack, J.P. Mannaerts, J. Kwo, S.N.G. Chu, N. Moriya, S.Y. Hou, V.J. Fratello, J. Vac. Sci. Technol. B14 (1996) 2297.

[11] M. Passlack, M. Hong, J.P. Mannaerts, J. Kwo, R.L. Opila, S.N.G. Chu, N. Moriya, F. Ren, IEEE Trans. Electron. Devices 44 (1997) 214

[12] M. Hong, Z.H. Lu, J. Kwo, A.R. Kortan, J.P. Mannaerts, J.J. Krajewski, K.C. Hsieh, L.J. Chou, K.Y. Cheng, Appl. Phys. Lett. 76 (3) (2000) 312.

[13] H.C. Casey, Jr, M.B. Panish, Heterostructure Lasers, Part B, Academic Press, London, 1978.

[14] P.S. Dutta, K.S. Sangunni, H.L. Bhat, V. Kumar, J. Cryst. Growth 141 (1994) 44. 